\title{
Comparative Analysis of the Efficacy and Safety of Oxaliplatin Plus 5-Fluorouracil/Leucovorin (Modified FOLFOX6) with Advanced Gastric Cancer Patients having a Good or Poor Performance Status
}

\author{
Ilhan Hacibekiroglu1*, Hilmi Kodaz ${ }^{1}$, Bulent Erdogan', Esma Turkmen ${ }^{1}$, Asim \\ Esenkaya $^{2}$, Sernaz Uzunoglu ${ }^{1}$, Irfan Cicin ${ }^{1}$
}

\begin{abstract}
Background: Combination chemotherapy of 5 fluorouracil (5-FU) and leucovorin (LV) with oxaliplatin, mainly FOLFOX regimens, has shown considerable antitumor activity and a tolerable toxicity profile in gastric cancer. The goal of this study was to retrospectively compare the efficacy and toxicity of modified FOLFOX-6 (mFOLFOX6) regimen in advanced gastric cancer (AGC) patients with good and poor performance status (PS). Materials and Methods: AGC patients receiving the mFOLFOX6 regimen including oxaliplatin $85 \mathrm{mg} / \mathrm{m}^{2}$, bolus of $5-\mathrm{FU} 400 \mathrm{mg} / \mathrm{m}^{2}$ and $\mathrm{LV} 400 \mathrm{mg} / \mathrm{m}^{2}$ on the first day, followed by $2400 \mathrm{mg} / \mathrm{m}^{2}$ of $5-\mathrm{FU}$ as a continious infusion over 46 hour for first-line treatment were eligible for the study. Results: A total 58 patients with a median age of $59.5(32-81)$ were included. The median follow up of the study was 9.2 months. Thirty patients $(51.7 \%)$ with an ECOG PS 0-1 were assigned to the good PS arm, while 28 patients $(48.3 \%)$ with ECOG PS 2 were in the poor PS arm. Overall response rates were 36.6 and $28.8 \%$, respectively ( $p=0.91$ ). Median PFS was 6.7 and 6.3 months in good PS and poor PS arms ( $p=0.50$ ) and median OS was 9.6 and 10.4 months ( $p=0.55)$. As compared with good PS arm, poor PS arm was associated with more grade 3-4 neutropenia and anemia. Dose reduction and dose delays were also significantly higher. Conclusions: In this study, mFOLFOX6 was similarly effective in both arms. Although hematologic toxicity was significantly higher in patients with poor PS, it remained manageable. Our results suggest that this regimen may be an effective treatment option for AGC patients with poor PS.
\end{abstract}

Keywords: First line - FOLFOX - gastric cancer - performance status

Asian Pac J Cancer Prev, 16 (6), 2355-2359

\section{Introduction}

Gastric cancer is the third leading cause of cancerrelated death worldwide (Ferlay et al., 2014). Despite the reduced incidence and mortality of gastric cancer over the past two decades, more than 40 percent of patients with gastric cancer are diagnosed with metastatic disease (Siegel et al., 2014). Advanced gastric cancer (AGC) usually has a poor prognosis and a five-year survival rate is below 5\% (Chang et al., 2012). Palliative chemotherapy can improve survival and quality of life compared to best supportive care in several randomized trials, therefore it is offered as a routine treatment option to patients with sufficient performance status (PS) (Pyrhonen et al., 1995; Glimelius et al., 1997). A metaanalysis showed that combination chemotherapy provides significant survival benefit, especially with three drugs combination, compared to single-agent chemotherapy (Wagner et al., 2006).
Although many different combination chemotherapies such as epirubicin, cisplatin and 5 fluorouracil (ECF), docetaxel, cisplatin and 5-FU (DCF) are used as standard treatment in Europe and The United States, globally accepted chemotherapy regimen still does not exist due to concerns for the toxicity and inconsistency in responses to treatment (Bilici et al., 2015). Therapeutic trend is toward new treatment options with improved clinical efficacy and more acceptable toxicity profile.

The third generation platinum, oxaliplatin has synergistic activity with 5 FU even in FU resistant cell lines (Bleiberg and de Gramont, 1998). In numerous phase II studies, combination chemotherapy of $5 \mathrm{FU}$ and leucovorin with oxaliplatin, in different doses and schedules, mainly FOLFOX regimens, have been shown considerable antitumor activity and tolerable toxicity in AGC patients (Al-Batran et al., 2004; De Vita et al., 2005; Lordick et al., 2005; Sumpter et al., 2005). 
Many patients with AGC have poor PS and comorbidity. Poor PS is an unfavorable prognostic factor for many cancer types and it is usually associated with reduced response to chemotherapy and reduced survival (Miller, 1989). Additionally, patients with poor PS were excluded in most of the clinical trials, and treatment options are highly limited for such patients. Therefore, it is substantial to determine an optimal treatment option for these patients.

To address this issue, we conducted a retrospective analysis to compare treatment efficacy and toxicity of mFOLFOX6 regimen in AGC patients with good PS and poor PS .

\section{Materials and Methods}

AGC patients histologically diagnosed between February 2011 and March 2014 were retrospectively analyzed. Patients who received modified FOLFOX6 (mFOLFOX6) regimen as first line treatment for recurrent gastric cancer after initial curative resection or presented with metastatic disease were included to the study. The mFOLFOX6 protocol including oxaliplatin $85 \mathrm{mg} / \mathrm{m}^{2}$ and $\mathrm{LV} 400 \mathrm{mg} / \mathrm{m}^{2}$ were given as a 2 hour intravenous infusion, followed by a $5 \mathrm{FU}$ bolus of $400 \mathrm{mg} / \mathrm{m} 2$ administered as a $10 \mathrm{~min}$ infusion and followed by $5 \mathrm{FU} 2400 \mathrm{mg} /$ $\mathrm{m}^{2}$ as a continuous infusion over 46 hours. The cycles were repeated every 2 weeks and the chemotherapy was continued until disease progression, unacceptable toxicity, the patient refusal or physician decision. Demographic, medical and toxicity data were obtained from the medical charts and chemotherapy charts. This retrospective study was approved by the institutional review board at Trakya University.

Performance status of the patients were determined according to ECOG (Eastern Cooperative Oncology Group) performance status scale. Patients were classified to two arm according to ECOG PS as good PS arm and poor PS arm. Patients who had ECOG PS 0 or 1 were included good PS arm, and patients who had ECOG PS 2 were included poor PS arm.

Response evaluation was performed every 4-6 cycles according to Response Evaluation Criteria in Solid Tumors (RECIST) guidelines, version 1.1 (Eisenhauer et al., 2009). Adverse Events were graded according to the National Cancer Institute's Common Terminology Criteria for (CTCAE), version 4.0 (Cirillo et al., 2009).

The time to progression (TTP) was measured from initiation of treatment until first evidence of progression, and the overall survival (OS) was measured from the initiation of treatment until death or last control date. If a patient died due to presumed progressive disease, in the absence of radiographic evidence of progression, the death date was used as the date of progression.

\section{Statistical analysis}

Baseline characteristics of good PS and poor PS arms

Table 1. Patient's Characteristics

\begin{tabular}{|c|c|c|c|c|c|}
\hline & \multicolumn{2}{|c|}{ Good PS arm $(n=30)$} & \multicolumn{2}{|c|}{ Poor PS arm $(n=28)$} & \multirow[t]{2}{*}{ p-value } \\
\hline & $\mathrm{n}$ & $(\%)$ & $\mathrm{n}$ & $(\%)$ & \\
\hline \multicolumn{6}{|l|}{ Sex } \\
\hline Male & 19 & $(63.3)$ & 25 & $(89.3)$ & 0.02 \\
\hline Female & 11 & $(36.7)$ & 3 & $(10.7)$ & \\
\hline Age. Median. Range & 54.4 & $(33-80)$ & 63 & $(32-80)$ & 0.006 \\
\hline Aged over 65 years & 6 & $(20.0)$ & 13 & $(46.4)$ & 0.03 \\
\hline \multicolumn{6}{|l|}{ Disease status } \\
\hline Locally advanced & 3 & (10) & 2 & $(7.1)$ & 0.70 \\
\hline Metastatic & 27 & (90) & 26 & (92.9) & \\
\hline Newly diagonized & 24 & (80) & 22 & $(78.6)$ & 0.90 \\
\hline Recurrent & 6 & (20) & 6 & $(21.4)$ & \\
\hline \multicolumn{6}{|l|}{ Tumor localization } \\
\hline Cardia-fundus & 9 & (30) & 10 & $(35.7)$ & 0.52 \\
\hline Corpus & 10 & $(33.3)$ & 11 & $(39.3)$ & \\
\hline Antrum & 9 & $(30)$ & 7 & (25) & \\
\hline Linitisplastica & 2 & $(6.7)$ & 0 & $(0)$ & \\
\hline \multicolumn{6}{|l|}{ Surgical history } \\
\hline Primary surgery & 8 & $(42.1)$ & 9 & $(56.2)$ & 0.40 \\
\hline Any palliative surgery & 11 & $(57.9)$ & 7 & $(43.8)$ & \\
\hline \multicolumn{6}{|l|}{ Adjuvant treatment } \\
\hline No & 24 & $(80)$ & 22 & $(78.6)$ & 0.90 \\
\hline Yes & 6 & (20) & 6 & $(21.4)$ & \\
\hline \multicolumn{6}{|c|}{ No. of organs involved in metastatic disease } \\
\hline 0 & 3 & $(10)$ & 2 & $(7.1)$ & 0.85 \\
\hline 1 & 17 & $(56.7)$ & 15 & $(53.6)$ & \\
\hline$>2$ & 10 & $(33.6)$ & 11 & $(39.3)$ & \\
\hline \multicolumn{6}{|c|}{ Organs most commonly involved } \\
\hline Liver & 11 & $(36.7)$ & 17 & $(60.7)$ & 0.07 \\
\hline Peritoneum & 12 & $(40.0)$ & 4 & $(14.3)$ & 0.03 \\
\hline Lung & 8 & (26.7) & 11 & (39.3) & 0.30 \\
\hline Bone & 2 & $(6.7)$ & 3 & $(10.7)$ & 0.60 \\
\hline
\end{tabular}


Efficacy and Safety of Modified FOLFOX6 for Advanced Gastric Cancer Patients with Good or Poor Performance Status.

were compared by $\mathrm{X}^{2}$ tests (for categorical variables) or two independent sample $t$ test (for continuous variables). The Kaplan-Meier method was used to provide median point estimates, TTP and median OS, and confidence intervals (CIs) calculated by Greenwood's formula. The log rank test was used to examine the statistical significance of differences demonstrated between the groups. Survival curves were created with SPSS software version 18.0 (SPSS, Chicago, IL). Safety analyses were performed by using descriptive statistics. P values of $<0.05$ were considered statistically significant.

\section{Results}

Fifty-eight patients were enrolled in this study. The median follow up of the study was 9.2 (2.7-25.3) months. Twelve patients had recurrent gastric cancer, and 46 patients were newly diagnosed0020AGC. Thirty patients (51.7\%) with ECOG PS 0-1 were included in good PS arm, while 28 patients $(48.3 \%$ ) with ECOG PS 2 were included in poor PS arm. The median age of the patients was 59.5 (32-81) years, whereas it was 54.4 (33-80) years in good PS and 63(32-80) years in poor PS groups, respectively $(p=0.006)$. Proportion of elderly patients aged over 65 years was significantly higher in poor PS arm than good PS arm $(p=0.03)$. Baseline characteristics of patients are summarized in Table 1. A total of 503 cycles were delivered, with a median number of 9 cycles per patient (range, 2-12).

Overall response rate (ORR) was $36.6 \%$ and $28.8 \%$ of patients who had good PS and poor PS arms, respectively $(\mathrm{p}=0.91)$. Median TTP was 6,7 (95\%CI, 2.4-10.9) and 6.3 (95\%CI, 5.7-6.8) months in good PS and poor PS arms, respectively (Figure $1, \mathrm{p}=0.50$ ). Median OS was 9.6 (95\%CI, 1.7-17.5) and 10.4 (95\%CI, 5.9-14.8) months in good PS and poor PS arms, respectively (Figure 2, $\mathrm{p}=0.55)$.

The most commonly observed grade 3-4 hematologic toxicity was neutropenia and anemia in both arms. As compared with good PS arm, poor PS arm had more grade 3-4 neutropenia and anemia. One patient experienced febrile neutropenia in good PS arm. The most common encountered grade 3-4 non-hematologic toxicity was nausea-vomiting, mucositis and diarrhea in both arms. No difference in non-hematologic toxicity was found in both arms. Grade 3-4 peripheral neuropathy was observed in $1(3.3 \%)$ patients and $2(7.1 \%)$ patients in good PS and poor PS arms, respectively $(\mathrm{p}=0.02)$. Toxicities observed during the treatment are summarized in Table 2. Dose was reduced in $10(33.3 \%)$ patients and $18(64.3 \%)$ patients in good PS and poor PS arms, respectively $(\mathrm{p}=0.02)$. Dose delay was occurred in $13(43.3 \%)$ patients and $20(71.4 \%)$ patients in good PS and poor PS arms, respectively $(\mathrm{p}=0.03)$. Treatment was discontinued in $2(6.7 \%)$ patients and $4(14.3 \%)$ patients in good PS and poor PS arms, respectively $(\mathrm{p}=0.34)$. Treatment related death occurred in just one patient in good PS arm.

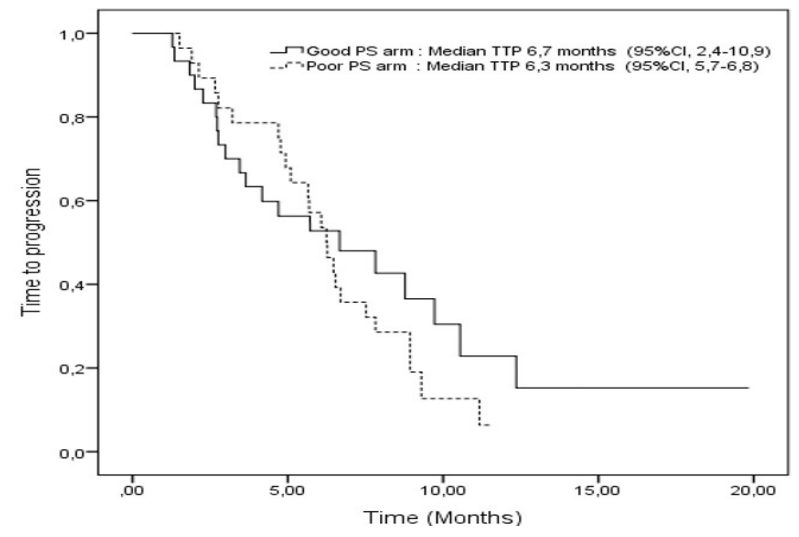

Figure 1. Time to Progression (TTP) Curves According to Performance status (PS)

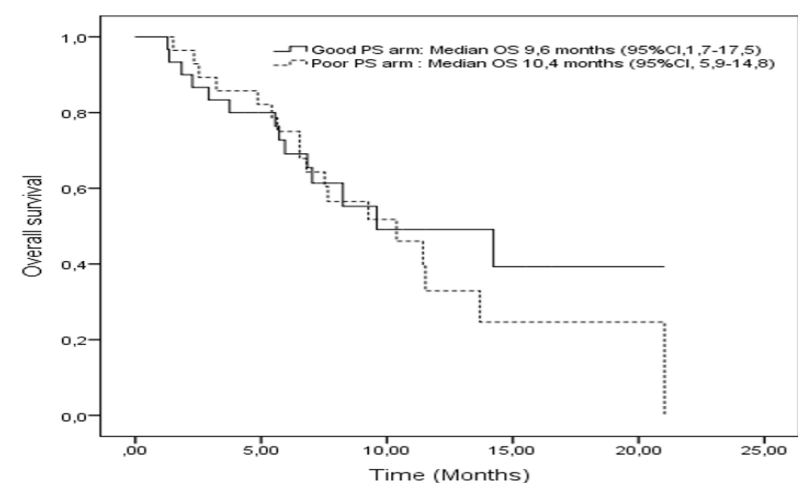

Figure 2. Overall Survival (OS) Curves According to Performance Status (PS)

Table 2. Toxicities According to NCI-CTC Version 2.0

\begin{tabular}{|c|c|c|c|c|c|}
\hline \multirow[t]{3}{*}{ Grade 3-4 Adverse events } & \multicolumn{4}{|c|}{ ECOG PS } & \multirow[t]{3}{*}{ P-value } \\
\hline & \multicolumn{2}{|c|}{ Good PS arm } & \multicolumn{2}{|c|}{ Poor PS arm } & \\
\hline & $\mathrm{n}=30$ & $\%$ & $\mathrm{n}=28$ & $\%$ & \\
\hline \multicolumn{6}{|l|}{ Non-hematolojical toxicities } \\
\hline Nausea - vomiting & 3 & $(10)$ & 5 & $(17.9)$ & 0.38 \\
\hline Diarrhea & 1 & $(3.3)$ & 3 & $(10.7)$ & 0.27 \\
\hline Mucositis & 2 & $(6.7)$ & 3 & $(10.7)$ & 0.58 \\
\hline Peripheral neuropathy & 1 & $(3.3)$ & 2 & $(7.1)$ & 0.51 \\
\hline \multicolumn{6}{|l|}{ Hematolojical toxicities } \\
\hline Neutropenia & 9 & $(30)$ & 16 & $(57.1)$ & 0.037 \\
\hline Febrile neutropenia & 0 & $(0)$ & 1 & (3.6) & 0.30 \\
\hline Anemia & 3 & (10) & 9 & $(32.1)$ & 0.038 \\
\hline Thrombocytopenia & 2 & $(6.7)$ & 3 & $(10.7)$ & 0.60 \\
\hline
\end{tabular}

*NCI-CTC: National cancer institute- common toxicity criteria, PS: performance status 


\section{Discussion}

In recent years, although the proportion of patients receiving palliative chemotherapy has increased dramatically, and new chemotherapeutic agents have been tested in AGC, it did not lead to an increase in overall survival in patients with AGC (Bernards et al., 2013) (Liu et al., 2014). Several phase 2 trials conducted in recent years have indicated that FOLFOX6 regimen is an effective and well tolerated treatment option for patients with AGC. Chemotherapy is offered as the standard treatment to increase survival and improve quality of life of patients with AGC. Treatment options are limited in patients who have poor PS, because they have been excluded in most of the clinical trials. Therefore, it is substantial to determine an optimal treatment option for such patients. Thus, we aimed to evaluate the tolerability and efficiency of FOLFOX chemotherapy in AGC patients with good and poor PS.

This study showed that the efficacy of mFOLFOX6 regimen among AGC patients with poor PS is not inferior to the efficacy among AGC patients with poor PS in terms of ORR, TTP and overall survival. Additionally, although grade 3-4 hematologic toxicities were significantly higher in patients with poor PS, toxicities were manageable and treatment compliance was good.

Poor PS is an unfavorable prognostic factor for many cancer types and it is usually associated with reduced response rate and shorter survival (Miller, 1989). According to meta-analysis of three phrase- 3 trials conducted between 1992-2001, patients with ECOG PS 2 was represented $22.8 \%$ of all patients and these patients experienced significantly poorer survival compared to patients with more favorable PS (Chau et al., 2004). Few PS 2 patients $(0 \%-10 \%)$ were included in recent phase III trials, therefore, standard treatment for patients with PS 2 has not been clearly determined yet (Shitara et al., 2009).

The effectiveness of a variety of FOLFOX6 regimens in AGC treatment has been evaluated in several studies. In general, ORR were $40.2 \%-48 \%$, TTP was 5,4-6,2 months and OS was 8,6-13 months (Louvet et al., 2002; Al Batran et al., 2004; Cavanna et al., 2006; Hwang et al., 2008; Luo et al., 2008; Lee et al., 2010). However, due to low number of patients enrolled in the studies have restricted the definitive conclusions.

Shitara et al. (2009) retrospectively analyzed AGC patients to evaluate efficiency of first line treatments according to PS. In this analysis, the rate of PS 2 patients was $17,0 \%$ and both TTF and OS were significantly longer in patients with PS 0 and 1 than with patients with PS 2 . Kim et al. administered FOLFOX 6 to 23 AGC patients with ECOG PS 2. In this study, ORR was $31.8 \%$, median TTP and OS were 3.5 and 9.2 months, respectively (de Gramont et al., 2000) (Kim et al., 2012). In our study, $48.3 \%$ of all the patients had ECOG PS 2, and we compared effectiveness of mFOLFOX6 between patients with poor and good PS. In our study, ORR was 36,6\% and $28.6 \%$ in good PS and poor PS arms, respectively. Median PFS was 6,7 and 6,3 months, whereas median OS was 9,6 and 10,4 months in good PS and poor PS arms, respectively. In terms of results, there was no significant difference between good and poor PS arms. ORR, median TTP and OS in both arms of our study were comparable to results yielded from previous studies. While ORR was lower than previous studies, our study had higher rates of stable disease with lower disease progression.

In our study, both good and poor PS arms had similar TTP, OS and ORR with different toxicity profile. In respect to toxicity, mFOLFOX6 regimen had manageable toxicity profile. Patient compliance was good. In poor PS arm, grade 3-4 neutropenia and anemia were significantly higher compared to good PS arm. In previousstudies with FOLFOX6 regimens for AGC, grade 3-4 neutropenia, anemia and thrombocytopenia were reported 4.9-34.1, $1.2-20 \%$ and $0-7.3 \%$ respectively (Al Batran et al., 2004; Louvet et al., 2002; Cavanna et al., 2006; Hwang et al., 2008; Luo et al., 2008; Lee et al., 2010). Incidence of grade 3-4 adverse effects in patients with good PS was similar to previous studies. Dose reduction and dose delays were significantly higher in poor PS arm. In terms of grade 3-4 non-hematologic toxicity, there was no significant difference between two groups.

High dose oxaliplatin $(100 \mathrm{mg} / \mathrm{m} 2)$ is associated with improved response rate, TTP and also increased incidence of neuropathy in the treatment of advanced colorectal cancer (de Gramont et al., 2000). In our study, we administered oxaliplatin $85 \mathrm{mg} / \mathrm{m} 2$ to avoid neuropathy. Louvet et al. reported higher incidence of grade 3 neuropathy $(21 \%)$ with FOLFOX6 regimen including oxaliplatin $100 \mathrm{mg} / \mathrm{m} 2$ than other studies (Louvet et al., 2002). In our study, grade 3 neuropathy was observed in only $3(5.2 \%)$ of all the patients. This proportion was similar to that of FOLFOX regimens administering oxaliplatin $85 \mathrm{mg} / \mathrm{m}^{2}$.

The study had certain limitation related to retrospective design and indirect comparison. Firstly, PS is not a precise criterion for evaluating the general status of cancer patients. However, there is still no alternative available for classifying general status. The use of more extensive criteria including age, general status, comorbidity and nutritional status can allow better treatment decisions. Presence of adverse events data only about the grade 3-4 toxicity are another limitation of our study. We could not compared grade 1-2 toxicity.

In conclusion, mFOLFOX6 was similarly effective in both arms. Our results suggest that this regimen may be an effective treatment option for AGC patients with poor PS.

\section{References}

Al-Batran SE, Atmaca A, Hegewisch-Becker S, et al (2004). Phase II trial of biweekly infusional fluorouracil, folinic acid, and oxaliplatin in patients with advanced gastric cancer. $J$ Clin Oncol, 22, 658-63.

Bernards N, Creemers GJ, Nieuwenhuijzen GA, et al (2013). No improvement in median survival for patients with metastatic gastric cancer despite increased use of chemotherapy. Ann Oncol, 24, 3056-60.

Bleiberg H, de Gramont A (1998). Oxaliplatin plus 5-fluorouracil: clinical experience in patients with advanced colorectal cancer. Semin Oncol, 25, 32-9.

Bilici A, Selcukbiricik F, Demin N, et al (2015). Modified docetaxel and cisplatin in combination with capecitabine 
Efficacy and Safety of Modified FOLFOX6 for Advanced Gastric Cancer Patients with Good or Poor Performance Status.

(DCX) as a first-line treatment in HER2-negative advanced gastric cancer. Asian Pac J Cancer Prev, 15, 8661-6

Cavanna L, Artioli F, Codignola C, et al (2006). Oxaliplatin in combination with 5-fluorouracil (5-FU) and leucovorin (LV) in patients with metastatic gastric cancer (MGC). Am J Clin Oncol, 29, 371-5.

Chang YR, Han DS, Kong SH, et al (2012). The value of palliative gastrectomy in gastric cancer with distant metastasis. Ann Surg Oncol, 19, 1231-9.

Chau I, Norman AR, Cunningham D, et al (2004). Multivariate prognostic factor analysis in locally advanced and metastatic esophago-gastric cancer--pooled analysis from three multicenter, randomized, controlled trials using individual patient data. J Clin Oncol, 22, 2395-403.

Cirillo M, Venturini M, Ciccarelli L, et al (2009). Clinician versus nurse symptom reporting using the National Cancer InstituteCommon Terminology Criteria for Adverse Events during chemotherapy: results of a comparison based on patient's self-reported questionnaire. Ann Oncol, 20, 1929-35.

de Gramont A, Figer A, Seymour M, et al (2000). Leucovorin and fluorouracil with or without oxaliplatin as first-line treatment in advanced colorectal cancer. J Clin Oncol, 18, 2938-47.

De Vita F, Orditura M, Matano E, et al (2005). A phase II study of biweekly oxaliplatin plus infusional 5-fluorouracil and folinic acid (FOLFOX-4) as first-line treatment of advanced gastric cancer patients. Br J Cancer, 92, 1644-9.

Eisenhauer EA, Therasse P, Bogaerts J, et al (2009). New response evaluation criteria in solid tumours: revised RECIST guideline (version 1.1). Eur J Cancer, 45, 228-47.

Ferlay J, Soerjomataram I, Dikshit R, et al (2014). Cancer incidence and mortality worldwide: Sources, methods and major patterns in GLOBOCAN 2012. Int J Cancer.

Glimelius B, Ekstrom K, Hoffman K, et al (1997). Randomized comparison between chemotherapy plus best supportive care with best supportive care in advanced gastric cancer. Ann Oncol, 8, 163-8.

Hwang WS, Chao TY, Lin SF, et al (2008). Phase II study of oxaliplatin in combination with continuous infusion of 5-fluorouracil/leucovorin as first-line chemotherapy in patients with advanced gastric cancer. Anticancer Drugs, 19, 283-8.

Kim HS, Kim JH, Kim HJ, et al (2012). Oxaliplatin, 5-fluorouracil and leucovorin (modified FOLFOX-6) as first-line chemotherapy for advanced gastric cancer patients with poor performance status. Oncol Lett, 3, 425-8.

Lee HH, Hur H, Kim SH, et al (2010). Outcomes of modified FOLFOX-6 as first line treatment in patients with advanced gastric cancer in a single institution; retrospective analysis. Cancer Res Treat, 42, 18-23.

Liu J, Huang XE, Feng JF (2014). Further study on pemetrexed based chemotherapy in treating patients with advanced gastric cancer (AGC). Asian Pac J Cancer Prev, 15, 6587-90

Lordick F, Lorenzen S, Stollfuss J, et al (2005). Phase II study of weekly oxaliplatin plus infusional fluorouracil and folinic acid (FUFOX regimen) as first-line treatment in metastatic gastric cancer. Br J Cancer, 93, 190-4.

Louvet C, Andre T, Tigaud JM, et al (2002). Phase II study of oxaliplatin, fluorouracil, and folinic acid in locally advanced or metastatic gastric cancer patients. J Clin Oncol, 20 , 4543-8.

Luo HY, Xu RH, Zhang L, et al (2008). A pilot study of oxaliplatin, fluorouracil and folinic acid (FOLFOX-6) as first-line chemotherapy in advanced or recurrent gastric cancer. Chemotherapy, 54, 228-35.

Miller RJ (1989). The role of chemotherapy in the hospice patient. A problem of definition. Am J Hosp Care, 6, 19-26.

Pyrhonen S, Kuitunen T, Nyandoto P, et al (1995). Randomised comparison of fluorouracil, epidoxorubicin and methotrexate (FEMTX) plus supportive care with supportive care alone in patients with non-resectable gastric cancer. Br J Cancer, 71, 587-91.

Shitara K, Muro K, Matsuo K, et al (2009). Chemotherapy for patients with advanced gastric cancer with performance status 2. Gastrointest Cancer Res, 3, 220-4.

Siegel R, Ma J, Zou Z, et al (2014). Cancer statistics, 2014. CA Cancer J Clin, 64, 9-29.

Sumpter K, Harper-Wynne C, Cunningham D, et al (2005). Report of two protocol planned interim analyses in a randomised multicentre phase III study comparing capecitabine with fluorouracil and oxaliplatin with cisplatin in patients with advanced oesophagogastric cancer receiving ECF. Br J Cancer, 92, 1976-83.

Wagner AD, Grothe W, Haerting J, et al (2006). Chemotherapy in advanced gastric cancer: a systematic review and metaanalysis based on aggregate data. J Clin Oncol, 24, 2903-9. 